\title{
Laparoscopic Bladder-Preserving Surgery for Enterovesical Fistula Complicated with Benign Gastrointestinal Disease
}

\author{
Tsunekazu Mizushima Masataka Ikeda Mitsugu Sekimoto \\ Hirofumi Yamamoto Yuichiro Doki Masaki Mori \\ Department of Surgery, Osaka University Graduate School of Medicine, Suita, \\ Japan
}

\section{Key Words}

Intestinal fistulas - Surgical techniques - Minimally invasive surgery - Colorectal and small bowel · Diverticular disease · Crohn's disease

\begin{abstract}
Enterovesical fistula (EVF) is a relatively uncommon condition that is associated with severe morbidity. Minimally invasive and organ-preserving surgery should be performed in the case of EVF caused by benign diseases. We applied laparoscopic bladder-preserving surgery (LBPS) for EVF caused by benign gastrointestinal disease. Here, we report a surgical technique for LBPS. Patient and instrument port positioning are similar to those used in laparoscopic colorectal surgery. Dissection around the fistula is performed along the intestine as distant from the bladder as possible. If there is sufficient area around the intestinal portion of the fistula, it is isolated and resected using a linear stapler. If this approach is not possible, the intestinal fistula is sharply dissected as far away from the bladder as possible. LBPS for EVF was performed in 4 patients and included 3 direct sharp dissections and 1 stapling dissection. Three of the 4 patients did not require any further treatment for the bladder, and all procedures were feasibly accomplished under laparoscopic conditions. In conclusion, LBPS is feasible in cases of EVF caused by benign gastrointestinal disease, and we suggest that it should be the first choice of intervention in such cases.
\end{abstract}




\section{Introduction}

Enterovesical fistula (EVF) is a relatively uncommon condition; however, it is associated with a high incidence of morbidity. In the majority of cases, the symptoms of EVF are associated with a decrease in the patient's quality of life. It represents a rare complication of inflammatory or neoplastic diseases in the gastrointestinal and urinary tracts, but may also be caused by traumatic or iatrogenic injuries [1]. In cases of EVF caused by neoplastic disease, extensive surgery with multivisceral resection, including bowel resection and partial/total cystectomy, is needed. Conversely, for EVF caused by benign diseases, minimally invasive and organ-preserving surgery should be performed. We performed laparoscopic bladder-preserving surgery (LBPS) for EVF caused by benign gastrointestinal disease. We report a surgical technique and concept of LBPS for EVF caused by benign gastrointestinal disease. For a video of the procedure carried out in case 1 and case 2, see online supplementary material (www.karger.com/doi/10.1159/000339202).

\section{Surgical Techniques}

The diagnosis of EVF is not difficult. Most affected patients present with pathognomonic features of pneumaturia, fecaluria and recurrent urinary tract infections. A computed tomography scan can detect air in the bladder in the majority of patients. However, cystography, swallowing studies and enemas cannot be used to confirm the presence of a fistula. In order to minimize the likelihood of infection, surgery should be conducted after a course of antibiotic therapy or following total parenteral nutrition. Informed consent was obtained before operation in all cases. Patient and instrument port positioning are performed in a way similar to laparoscopic colorectal surgery.

First, malignancy is ruled out by laparoscopic examination. The relationship between the intestine, EVF and bladder is consequently confirmed. Then a mobilization approach of medial-to-lateral and lateral-to-medial is made for laparoscopic dissection and vessel ligation. Vessel ligation should be performed as distant from the root of the feeder arteries as possible. The identification and confirmation of the ureter must be ascertained and meticulous dissection is important for avoiding ureteral injury. Lymphadenectomy is not indicated because of the benign cause.

The fistula along the diseased intestine is dissected as distant from the bladder as possible. If isolation is not possible, the fistula is sharply dissected along the diseased intestine (fig. 1). If there is sufficient area around the intestinal fistula, it is isolated and resected using a linear stapler (fig. 2 ).

Following LBPS, the bladder is filled with saline (100-200 ml) to check for any leakage. In the event of a leak, curettage and suturing around the bladder fistula is undertaken to close the fistula. Neither partial cystectomy nor trimming of the bladder has further practical benefit. A Foley catheter is inserted into the bladder after the procedure.

Following fistula removal, the diseased intestine is resected and the tissue is reconstructed using stapling techniques (functional end-to-end anastomosis for the small intestine or right colon, and double-stapling technique for the left colon or rectum). The resected fistula site at the bladder is covered with omentum to prevent adhesion between the anastomotic site and the bladder. A drainage tube is inserted to assess any bleeding or urine leakage.

Postoperative oral intake is permitted as with conventional colorectal surgery. One week after the procedure, the Foley catheter is removed once cystography confirms the absence of any leakage from the bladder. 


\section{Results}

LBPS was performed on 4 patients with EVF caused by benign gastrointestinal disease. The demographics of the patients are given in table 1 . Median age was 48 years. Primary disease included diverticulitis of the sigmoid colon (3 patients) and Crohn's disease (1 patient). We employed the same surgical procedure in all cases, as described before. In the male patients with diverticulitis of the sigmoid colon, 2 direct sharp dissections and 1 stapling dissection of the fistula were performed. In the female patient with Crohn's disease, direct sharp dissection of the fistula was performed. The water leak test was positive in 1 patient after the direct sharp dissection, and this patient underwent suturing around the bladder fistula. The procedures for the diseased intestines included 3 sigmoidectomies with the double-stapling technique and 1 ileocecal resection with functional end-to-end anastomosis. The median operation time and blood loss were $234 \mathrm{~min}$ and $225 \mathrm{~g}$, respectively. All patients underwent cystography 1 week after the operation, and the Foley catheter was removed. The median postoperative follow-up period was 25 months. Neither dysuria nor recurrence of EVF was observed on follow-up.

\section{Discussion}

EVF is usually complicated by subsequent gastrointestinal diseases, such as diverticulitis, colorectal cancer and inflammatory bowel diseases. EVF caused by urologic disease is rare [2, 3]. EVFs caused by gastrointestinal diseases are mostly resistant to medical therapy and often require surgical correction. In cases of EVF caused by gastrointestinal malignancy, not only bowel resection with regional lymphadenectomy for the primary lesion, but also extensive surgery with multivisceral resection, such as total pelvic exenteration, is needed to complete en bloc resection. Construction of diverting stoma without bowel resection is common for palliation. In contrast, in cases of EVF caused by benign gastrointestinal disease, resection of the diseased portion of the intestine is fundamental to surgical treatment. However, a standard treatment for the bladder in patients with EVF caused by benign gastrointestinal disease has not been established.

Recent technological advances and development of new equipment have promoted the use of laparoscopic surgery as standard treatment for gastrointestinal diseases. The indications for laparoscopic surgery are expanding. However, laparoscopic surgery for internal fistula caused by benign gastrointestinal disease is still uncommon. Historically, partial cystectomy and formal repair of the bladder wall were performed as the typical treatment [4]. Therefore, the complicated procedures for bladder surgery, such as deciding upon an area and extent of resection or suturing a defect of the bladder wall, were considered difficult under laparoscopic surgery. Furthermore, partial cystectomy, if the area or extent of bladder resection is inadequate, may lead to dysuria. Successful surgical management of EVF from diverticulitis or Crohn's disease has recently been reported; the procedure involves only resection of the diseased bowel with minimal need for repair or resection of the bladder side of the fistula [5]. It is commonly believed that partial cystectomy should be considered only when major water leakage is detected after the repair of overt full-thickness defects in the bladder. During LBPS, we put this concept to practical use under laparoscopic surgery. The LBPS 
procedures carried out for EVF included 3 direct sharp dissections and 1 stapling dissection. The water leak test was positive in 1 patient who underwent direct sharp dissection, and this patient received subsequent suturing around the resection site to seal the bladder. In all patients the laparoscopic procedure was successfully performed. It cannot be denied that open conversion is necessary in cases with dense adhesion or with difficulties in identification and dissection of the ureter. However, we conclude that LBPS can be completed in most cases of EVF caused by benign gastrointestinal disease and should be the first choice of intervention.

Table 1. Patient characteristics

\begin{tabular}{lllll}
\hline & \multicolumn{2}{l}{ Case } & & \\
\cline { 2 - 5 } & 1 & 2 & 3 & 4 \\
\hline Age & 80 & 40 & 44 & 51 \\
Sex & male & male & male & female \\
Primary disease & diverticulitis & diverticulitis & diverticulitis & Crohn's disease \\
Enteric fistula & sigmoid colon & sigmoid colon & sigmoid colon & ileum \\
Bladder fistula & base & trigone & body & base \\
Fistula treatment & direct sharp & stapling & direct sharp dissection & direct sharp \\
& dissection & dissection & with additional suturing & dissection \\
Operating time, min & 325 & 235 & 233 & 100 \\
Blood loss, g & 190 & 200 & 520 & 250 \\
Complication & wound infection & none & none & none \\
\hline
\end{tabular}




\begin{tabular}{r|l|l|l}
$\begin{array}{r}\text { Case Reports in } \\
\text { Gastroenterology }\end{array}$ & $\begin{array}{l}\text { Case Rep Gastroenterol 2012;6:279-284 } \\
\text { DOI: 10.1159/000339202 }\end{array}$ & $\begin{array}{l}\text { Published online: } \\
\text { May 23, 2012 }\end{array}$ & $\begin{array}{l}\text { @ 2012 S. Karger AG, Basel } \\
\text { ISSN 1662-0631 } \\
\text { www.karger.com/crg }\end{array}$ \\
\hline
\end{tabular}

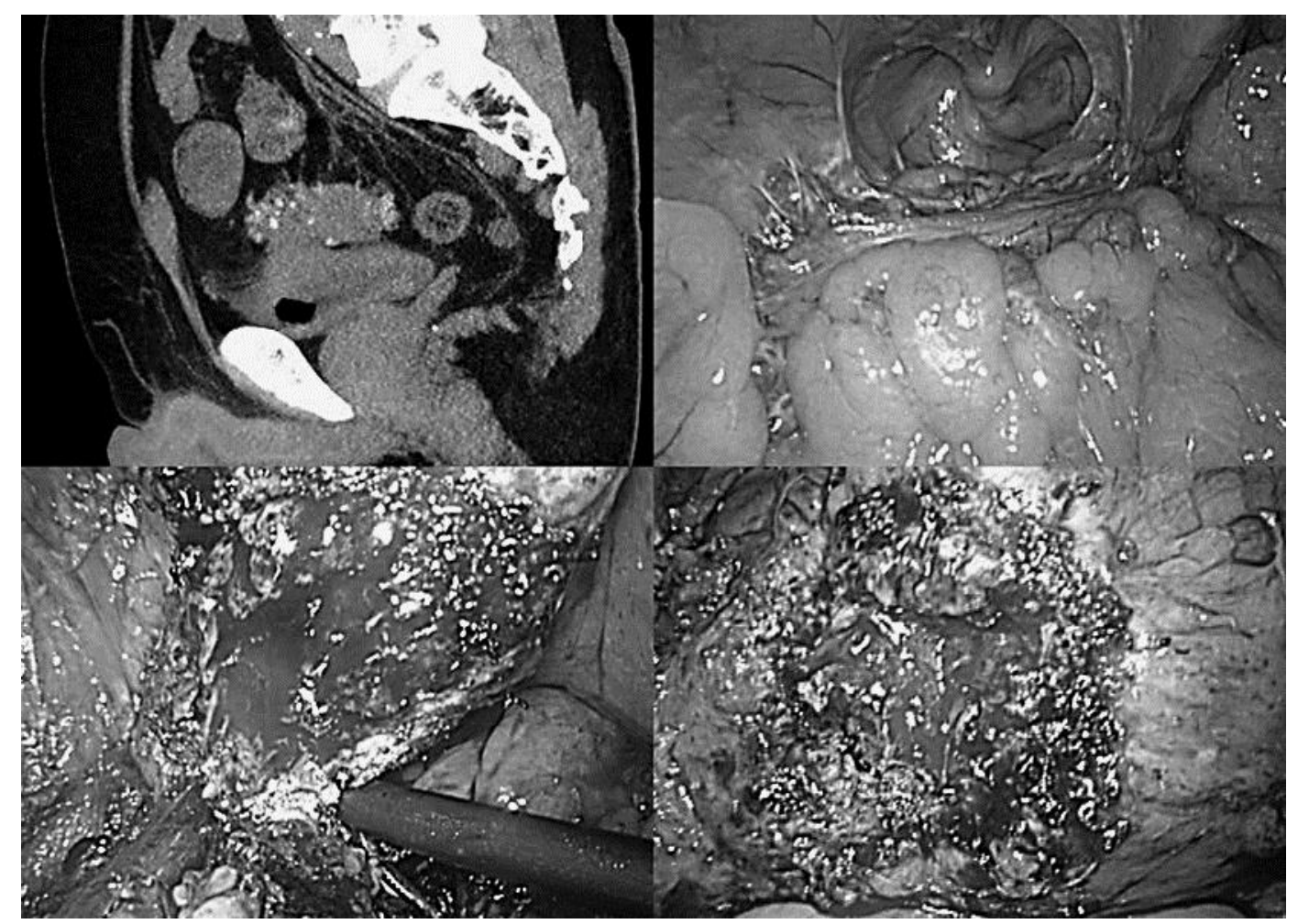

Fig. 1. Case 1. The patient was an 80 -year-old man with EVF caused by diverticulitis of the sigmoid colon. Bladder fistula was identified at the base. Severe inflammatory adhesion due to previous diverticulitis existed around the sigmoid colon. We dissected around the sigmoid colon and dissected the fistula sharply along the diseased intestine. The water leak test was negative, and hence no additional treatment for the bladder was needed. 


\begin{tabular}{r|l|l|l}
$\begin{array}{r}\text { Case Reports in } \\
\text { Gastroenterology }\end{array}$ & $\begin{array}{l}\text { Case Rep Gastroenterol 2012;6:279-284 } \\
\text { DOI: 10.1159/000339202 }\end{array}$ & $\begin{array}{l}\text { Published online: } \\
\text { May 23, 2012 }\end{array}$ & $\begin{array}{l}\text { @ 2012 S. Karger AG, Basel } \\
\text { ISSN 1662-0631 } \\
\text { www.karger.com/crg }\end{array}$ \\
\hline
\end{tabular}

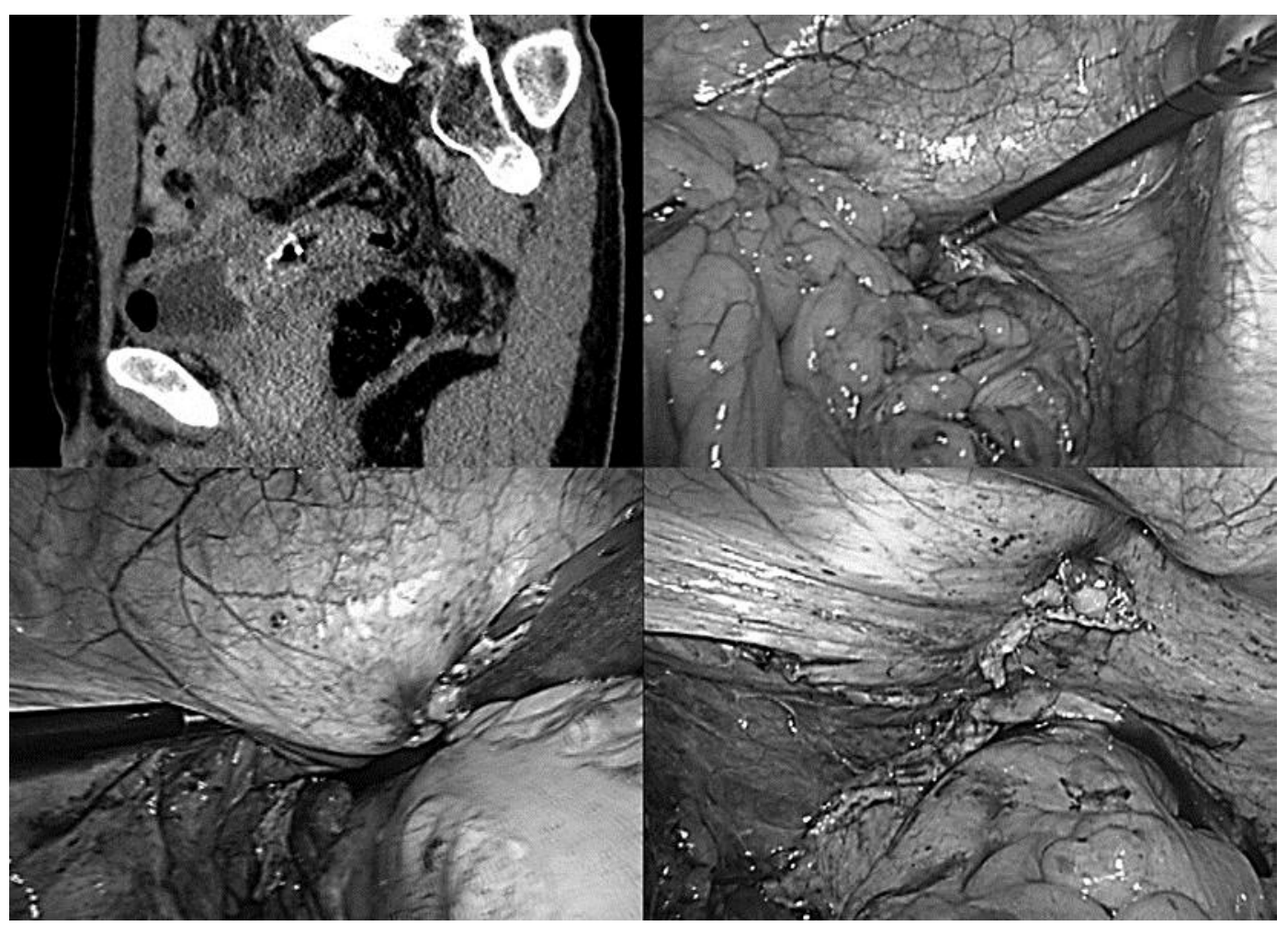

Fig. 2. Case 2. The patient was a 40-year-old man with EVF caused by diverticulitis of the sigmoid colon. Bladder fistula was identified at the trigone. The inflammatory adhesion around the fistula was very mild, and we could perform taping around the fistula. Thus, we identified the ureter and performed a stapling dissection of the EVF.

\section{References}

1 Kavanagh D, Neary P, Dodd JD, Sheahan KM, O’Donoghue D, Hyland JM: Diagnosis and treatment of enterovesical fistulae. Colorectal Dis 2005;7:286-291.

$\checkmark 2$ Couris GD, Block MA: Intestinovesical fistula. Surgery 1963;54:736-742.

3 Carson CC, Malek RS, Remine WH: Urologic aspects of vesicoenteric fistulas. J Urol 1978;119:744-746.

4 Rames RA, Bissada N, Adams DB: Extent of bladder and ureteric involvement and urologic management in patients with enterovesical fistulas. Urology 1991;38:523-525.

5 Ferguson GG, Lee EW, Hunt SR, Ridley CH, Brandes SB: Management of the bladder during surgical treatment of enterovesical fistulas from benign bowel disease. J Am Coll Surg 2008;207:569-572. 